Supporting Information

\title{
Naturally Extracted Hydrophobic Solvent and Self-Assembly in Interfacial Polymerization
}

Gheorghe Falca, $\stackrel{+}{+}$ Valentina E. Musteata, $\stackrel{+}{+}$ Stefan Chisca, $\stackrel{+}{*}$ Mohamed Nejib Hedhili, ${ }^{\dagger}$ Chisiang Ong, ${ }^{t}$ Suzana P. Nunes*t.

King Abdullah University of Science and Technology, Biological and Environmental Science

Engineering Division (BESE), Advanced Membranes and Porous Materials Center, 23955-6900

Thuwal, Saudi Arabia

'King Abdullah University of Science and Technology, Imaging and Characterization Laboratory, 23955-6900 Thuwal, Saudi Arabia 


\section{Corresponding author}

*Suzana P. Nunes - Biological and Environmental Science Engineering Division (BESE), King Abdullah University of Science and Technology, 23955-6900 Thuwal, Saudi Arabia. ORCID 0000-0002-3669-138X, Email: suzana.nunes@kaust.edu.sa

\section{Co-Authors}

Gheorghe Falca - Biological and Environmental Science Engineering Division (BESE), King Abdullah University of Science and Technology, 23955-6900 Thuwal, Saudi Arabia.

Valentina E. Musteata - Biological and Environmental Science Engineering Division (BESE), King Abdullah University of Science and Technology, 23955-6900 Thuwal, Saudi Arabia.

Stefan Chisca - Biological and Environmental Science Engineering Division (BESE), King Abdullah University of Science and Technology, 23955-6900 Thuwal, Saudi Arabia.

Mohamed Nejib Hedhili - Imaging and Characterization Laboratory, King Abdullah University of Science and Technology, 23955-6900 Thuwal, Saudi Arabia.

Chisiang Ong - Biological and Environmental Science Engineering Division (BESE), King Abdullah University of Science and Technology, 23955-6900 Thuwal, Saudi Arabia. 

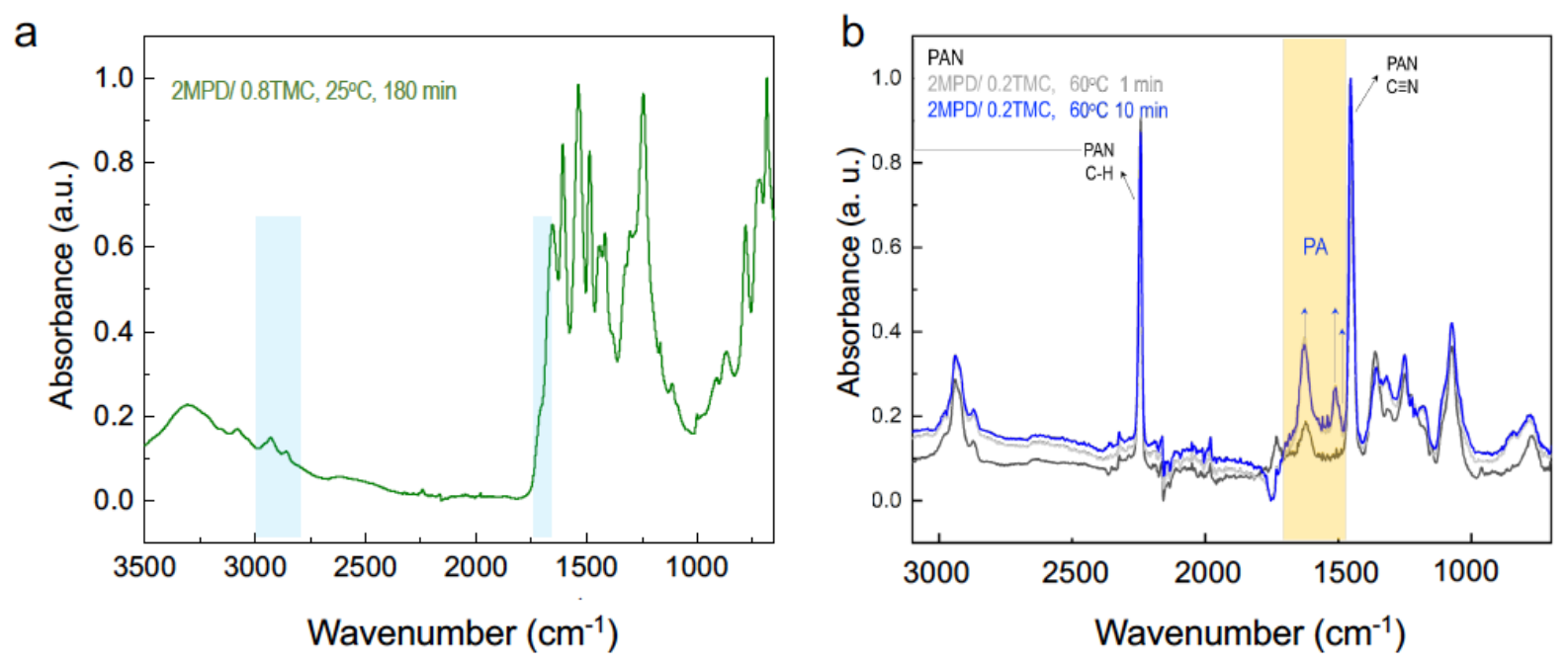

Figure S1. FTIR spectra of (a) thin-film composite membrane (on PAN support) prepared with 2 $\mathrm{wt} \% \mathrm{MPD}$ and $0.8 \mathrm{wt} \% \mathrm{TMC}$ at $25^{\circ} \mathrm{C}, 180 \mathrm{~min}$ as reaction time and washed in hexane, isopar and water for $72 \mathrm{~h}$, after preparation; (b) thin-film composite membranes (on PAN support) prepared with $2 \mathrm{wt} \% \mathrm{MPD}$ and $0.2 \mathrm{wt} \% \mathrm{TMC}$ at $60^{\circ} \mathrm{C}$ during 1 and $10 \mathrm{~min}$ as reaction times, as well as the spectrum of the plain PAN support. 

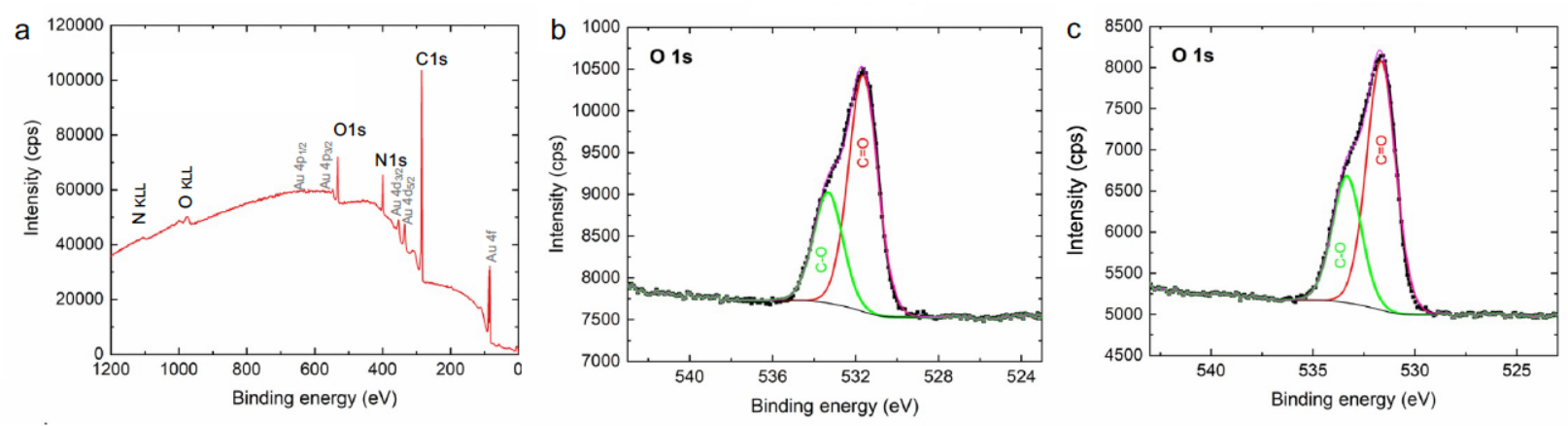

Figure S2. (a) XPS survey spectra of free-standing TFC membrane obtained using $2 \mathrm{wt} \% \mathrm{MPD} /$ $0.2 \mathrm{wt} \% \mathrm{TMC}$, and 1 minute reaction time; $(\mathrm{b}, \mathrm{c}) \mathrm{XPS}$ O1s spectra for the free-standing polyamide layer obtained using $2 \mathrm{wt} \% \mathrm{MPD} / 0.2 \mathrm{wt} \%$ TMC with (b) 1 and (c) 10 minutes as reaction time. 


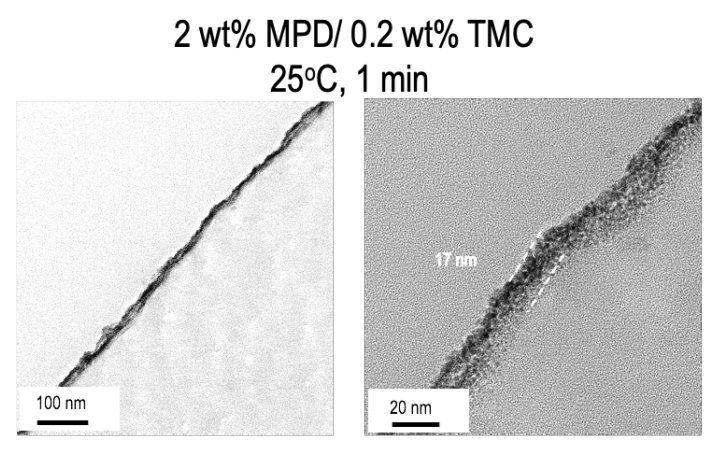

$2 w t \% \mathrm{MPD} / 0.2 \mathrm{wt} \% \mathrm{TMC}$

$60^{\circ} \mathrm{C}, 1 \mathrm{~min}$
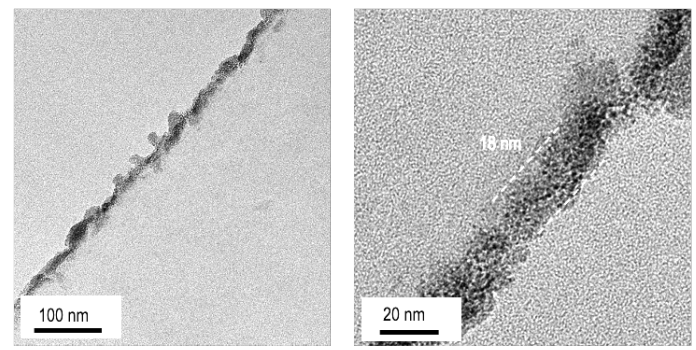

$2 w t \%$ MPD/ $0.2 w t \%$ TMC

$25^{\circ} \mathrm{C}, 10 \mathrm{~min}$

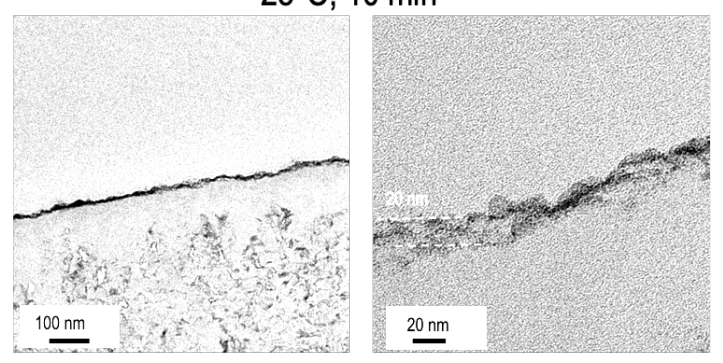

$2 w t \%$ MPD/ $0.2 w t \%$ TMC $60^{\circ} \mathrm{C}, 10 \mathrm{~min}$

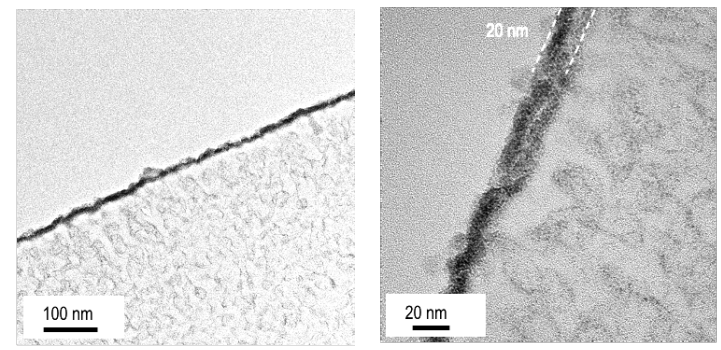

\section{$0.2 w t \%$ MPD/ 0.02 wt $\%$ TMC $25^{\circ} \mathrm{C}, 1 \mathrm{~min}$}
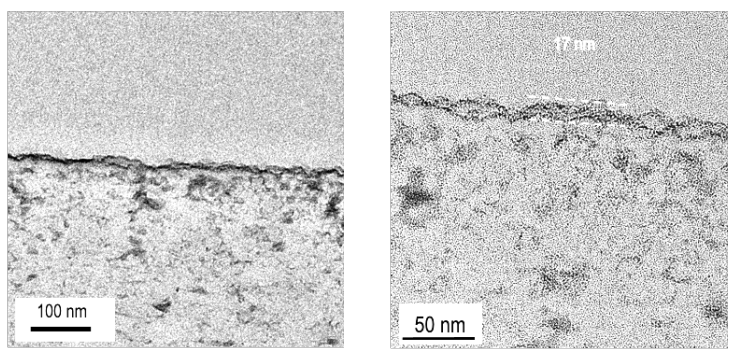

Figure S3. TEM cross-sectional image of ultrathin polyamide layer obtained with conditions reported in Table 1. 
$0.2 \mathrm{wt} \% \mathrm{MPD} / 0.2 \mathrm{wt} \% \mathrm{TMC} 180 \mathrm{~min}$

a

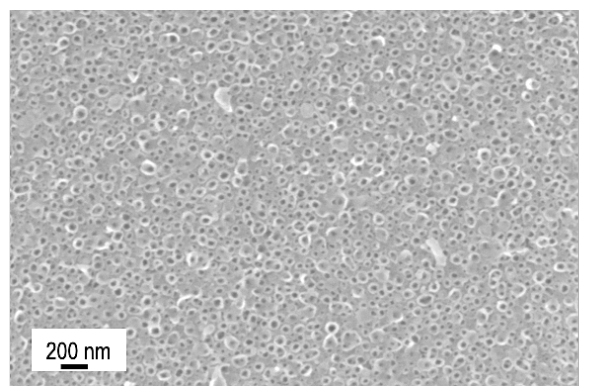

$0.2 \mathrm{wt} \% \mathrm{MPD} / 0.8 \mathrm{wt} \% \mathrm{TMC} 180 \mathrm{~min}$

b

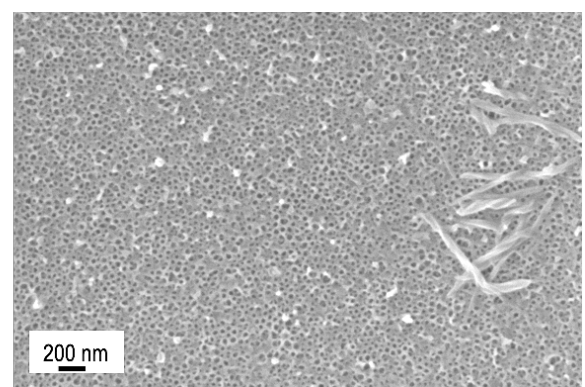

Figure S4. SEM images of the surface of membranes prepared with 180 minutes reaction time, with $0.2 \mathrm{wt} \%$ MPD and two different concentrations of TMC.

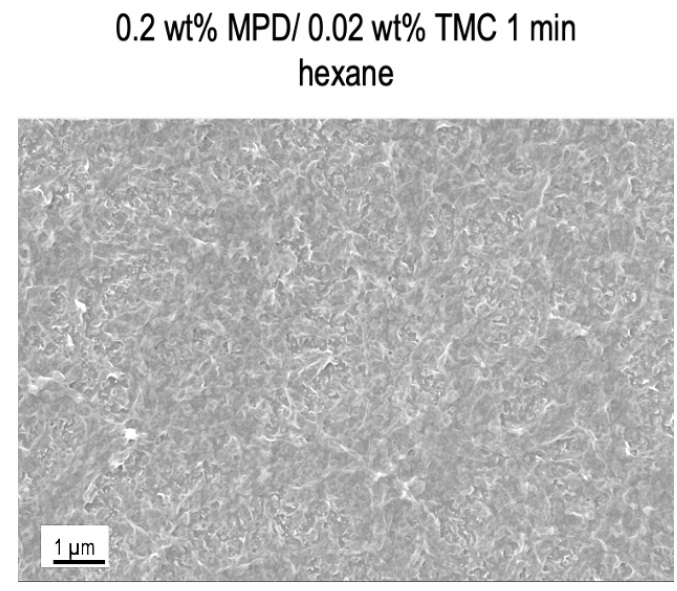

Figure S5. SEM surface image of the polyamide layer obtained with $0.2 \mathrm{wt} \% \mathrm{MPD}, 0.02 \mathrm{wt} \%$ TMC and 1 min reaction time in hexane. 

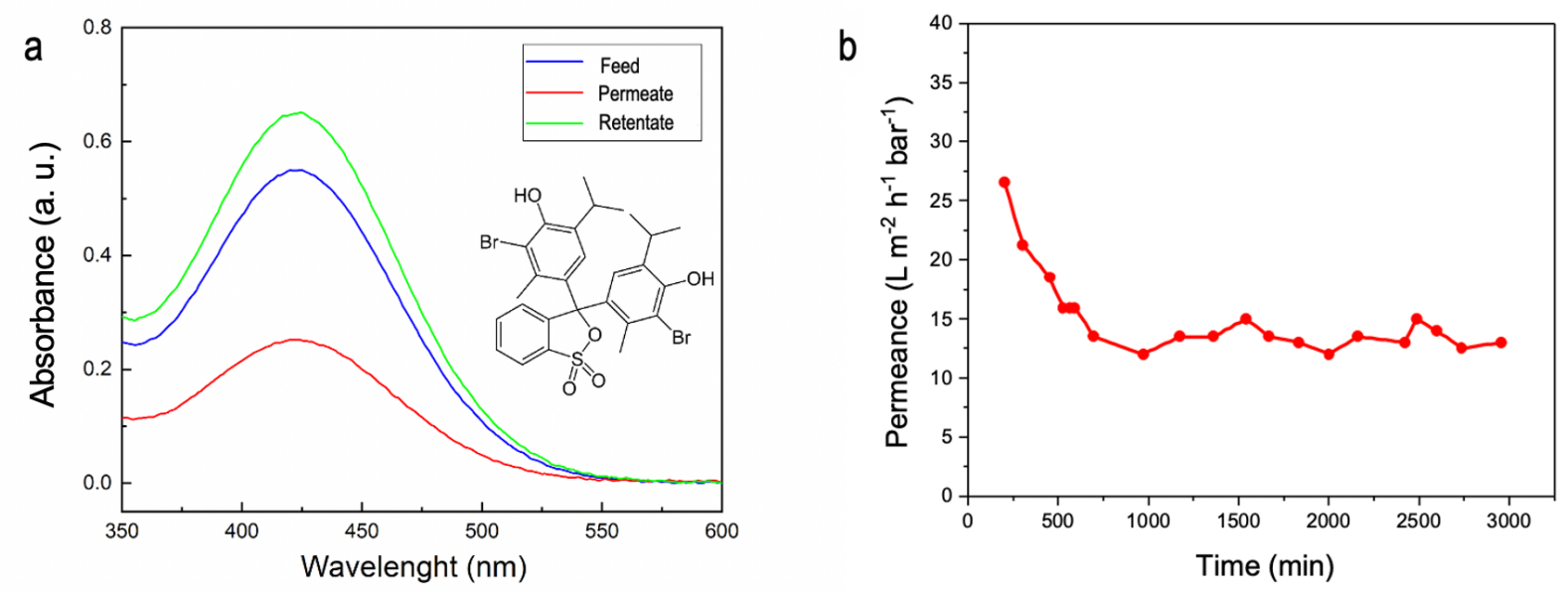

Figure S6. (a) UV spectra of feed, permeate and retentate, when testing membranes prepared with $0.2 \mathrm{wt} \% \mathrm{MPD} / 0.02 \mathrm{wt} \% \mathrm{TMC}$ using Bromothymol Blue as solute in methanol; (b) long-term permeance measured with solutions of Direct Red 80 in methanol as feed. 
Table S1. Dye structures and net total charge.

\begin{tabular}{|c|c|c|c|}
\hline Name & $\begin{array}{c}\mathrm{MW} \\
\left(\mathrm{g} \mathrm{mol}^{-1}\right)\end{array}$ & structure & $\begin{array}{c}\text { Net } \\
\text { charge }\end{array}$ \\
\hline Orange $\mathrm{G}$ & 452.4 & & -2 \\
\hline Bromothymol Blue & 624.4 & & 0 \\
\hline Reactive Blue 19 & 626.5 & & -2 \\
\hline Congo Red & 696.7 & & -2 \\
\hline Brilliant Blue R & 825.9 & & -2 \\
\hline Direct Red 80 & 1373 & tan & -6 \\
\hline Reactive Green 19 & 1418.9 & & -4 \\
\hline
\end{tabular}

